\title{
VUV Spectral Line Emission Measurements in the TCABR Tokamak
}

\author{
M.Machida \\ Instituto de Física, Universidade Estadual de Campinas-UNICAMP, Campinas, SP, 13083-970, Brazil \\ A.M.Daltrini \\ Centro de Excelência em Tecnologia Eletrônica Avançada - CEITEC, Porto Alegre, RS, Brazil
}

J.H.F.Severo, I.C.Nascimento, E.K.Sanada, J.I.Elizondo, Y.K.Kuznetsov
Instituto de Física, Universidade de São Paulo, São Paulo, SP, 05508-900, Brazil

(Received on 15 December, 2008)

\begin{abstract}
The study of tokamak plasma light emissions in the vacuum ultraviolet (VUV) region is an important subject since many impurity spectral emissions are present in this region. These spectral emissions can be used to determine the plasma ion temperature and density from different species and spatial positions inside plasma according to their temperatures. We have analyzed VUV spectra from $500 \AA$ to $3200 \AA$ wavelength in the TCABR tokamak plasma including higher diffraction order emissions. There have been identified 37 first diffraction order emissions, resulting in 28 second diffraction order, 24 third diffraction order, and 7 fourth diffraction order lines. The emissions are from impurity species such as OII, OIII, OIV, OV, OVI, OVII, CII, CIII, CIV, NIII, NIV, and NV. All the spectra beyond $1900 \AA$ are from higher diffraction order emissions, and possess much better spectral resolution. Each strong and isolated spectral line, as well as its higher diffraction order emissions suitable for plasma diagnostic is identified and discussed. Finally, an example of ion temperature determination using different diffraction order is presented.
\end{abstract}

Keywords: VUV spectrometry, higher diffraction order spectra, tokamak, ion temperature, multi-channel detector

\section{INTRODUCTION}

The line emissions from impurity particles in tokamak plasmas are routinely used in many analyses as radiation power loss, transport/rotation phenomena, and ion temperature measurements in different region of plasma [1-3]. In addition, many impurity emissions met in divertor or edge regions of large tokamak $[4,5]$ can be comparable to impurity emissions produced by plasmas in smaller tokamaks as TCABR [6].

The traditional forms to support these studies are the measurements in the VUV (vacuum ultraviolet) region of ion line Doppler broadening and/or shifting [7, 8], and effective charge calculations using calibrated absolute intensity measurements. However, in VUV region, the Doppler broadening is smaller than in the visible region (due to its wavelength), the absolute calibration is much more arduous, and measurable spectral range are smaller compared to the visible region [9].

Despite of many works in VUV region of tokamak plasmas, usually is difficult to find a list of most common spectral emissions, nearby emissions, and presence of higher diffraction order emissions. However, such as reported in a previous work [10], higher diffraction order emissions, which usually are ignored, can provide better measurement results, due to the direct relationship of diffraction order number with resolution power and angular dispersion. In this article, spectral lines from $500 \AA$ to $1900 \AA$ measured in TCABR tokamak plasmas are presented. First diffraction order spectra were detected up to $1900 \AA$, but several second, third, and fourth diffraction order emissions were also identified up to $3200 \AA$. An open micro channel plate (MCP) with charge coupled device (CCD) detector coupled to one-meter focal length VUV McPherson spectrometer has been used. The most important emissions, with their respective higher order lines, are listed and the usefulness of higher diffraction order emissions is an- alyzed.

\section{EXPERIMENTAL SETUP}

The VUV spectrometer is a 225 McPherson, which has one-meter focal length and $82.5^{\circ}$ incidence $\left(\sim 15^{\circ}\right.$ between the beam incident on the grating and a diffracted beam that will pass through the exit slit). It is provided with a 1200 groves/mm concave grating with $\mathrm{Al}$ and $\mathrm{MgF}_{2}$ coating, and blazed at $2000 \AA$. The spectrometer has the linear dispersion of $8,3 \AA / \mathrm{mm}$ and can cover the spectral region from $500 \AA$ to $3500 \AA$.

The multi-channel detector consists of an open $40 \mathrm{~mm}$ diameter MCP plate (BrightView XUV2010 G, XSI Instruments) coupled to a CCD device (Marconi CCD30-11, Andor Technology) with 1024 by 256 pixels, and each pixel has 26 micrometers by 26 micrometers. This device has been specially mounted for our spectrometer by McPherson Inc. [11]. The MCP plate is coated by CsI (to convert VUV photons in electrons), and has a phosphor screen to convert the electrons in photons. The MCP channels, separated by 12 micrometers, have 10 micrometers diameter. A reducing coherent glass fiber array was used to couple the MCP to the CCD; more detail can be found in the ref. [10].

The spectrometer is pumped below $1 \times 10^{-6}$ Torr by a 250 $\mathrm{L} / \mathrm{s}$ turbomolecular pump. It is connected to the tokamak chamber by a $3.6 \mathrm{~cm}$ diameter, 4.80 meter long stainless steel tube with differential pumping, and is aligned to observe the equatorial plane of the tokamak.

The main parameters of TCABR tokamak are [6]: major radius of $\mathrm{R}=0.615 \mathrm{~m}$, minor radius of $\mathrm{r}=0.18 \mathrm{~m}$, maximum toroidal magnetic field of $\mathrm{B}_{T}=1.1 \mathrm{~T}$, maximum plasma current of $\mathrm{I}_{p}=110 \mathrm{kA}$, and typical electron density and temperature of $2 \times 10^{19} \mathrm{~m}^{-3}$ and $400 \mathrm{eV}$ respectively.

The experiment was performed with hydrogen three staged 
gas puff injection during the discharge. All measurements were taken at $60 \mathrm{~ms}$ of the discharge, which corresponds to the plasma current of $90 \mathrm{kA}$ and line averaged central electron density of $1.2 \times 10^{19} \mathrm{~m}^{-3}$ as can be seen in Fig. 1 .
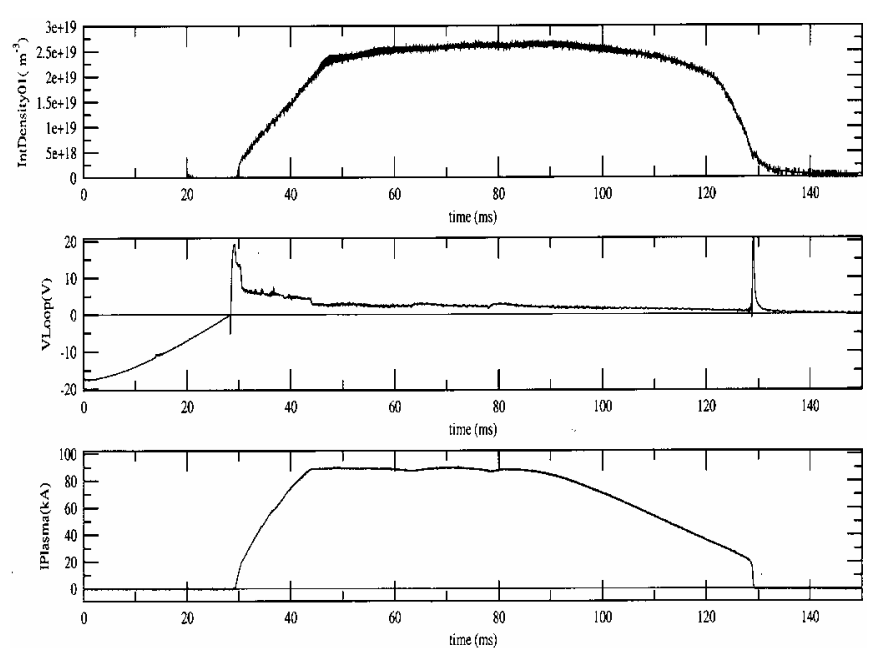

FIG. 1: Discharge signals from TCABR tokamak. From the top, electron density, loop voltage and plasma current. All the spectral measurements have been taken at discharge time of $60 \mathrm{~ms}$

The integration time for the spectral measurements, defined by MCP gate pulse, was taken as $5 \mathrm{~ms}$. The entrance slit of VUV spectrometer was kept at 80 microns.

Figure 2 shows the detector spectral response versus wavelength. The wavelengths were calibrated by different methods $[9,12]$, in order to cover a longer wavelength range. One can observe that wavelengths below $550 \AA$ and above $2300 \AA$ are much attenuated due to the detector sensitivity. Nevertheless, higher diffraction order emissions from the interval of $550 \AA$ to $1550 \AA$ still can be detected at higher wavelength positions since they kept the original wavelength.

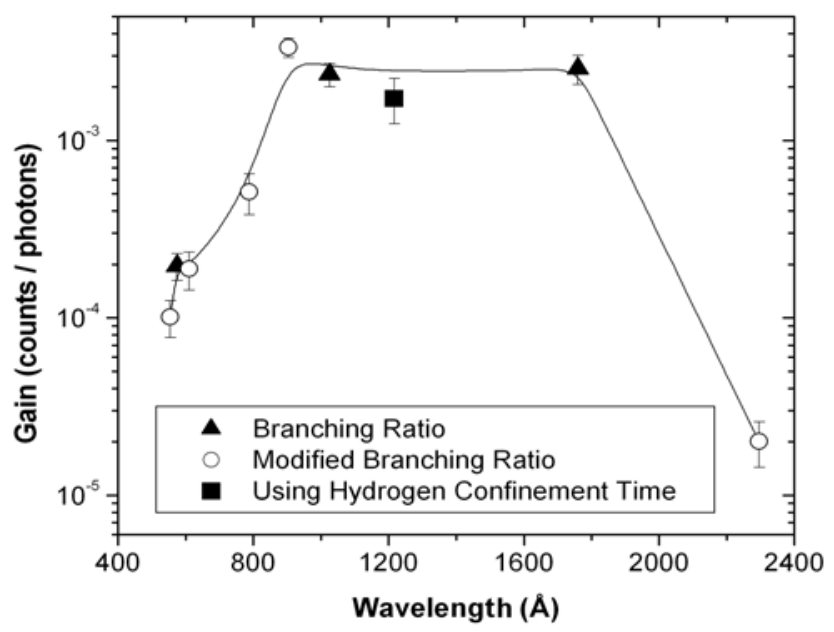

FIG. 2: Calibration points for the VUV spectrometer with multichannel detector using different calibration methods

\section{EXPERIMENTAL RESULTS AND DATA ANALYSIS}

The wavelength interval measured at each run is defined by 1024 pixels of the CCD. This corresponds to the wavelength interval of about $370 \AA$ for each tokamak discharge. From this interval, only central region of about $200 \AA$ has been used for our data analysis in order to avoid signal intensity decrease at the CCD edge pixels [10]. The measured spectrum was then plotted in Figs. 3 to 7 . For each figure, three (with exception of Fig. 6) TCABR tokamak discharges (indicated by the upper numbers in the central part of figures) were used in order to obtain an interval of about $500 \AA$. The number between parentheses for each spectral line is the same number as indicated in Table 1. The diffraction order number is indicated by number of asterisks with the specie element (asterisks were not used for first diffraction order lines). Since the measurement conditions were kept constant, by changing only the spectrometer wavelength interval, the relative intensity counts comparison between spectra is possible taking into account the spectrometer calibration curve seen in Fig. 2.

In Fig. 3 is presented the spectral emissions from $500 \AA$ to $1000 \AA$. Two strongest emissions are from OV at 629.73 $\AA$ (4) and CIII at $977.02 \AA$ (20), with 8296 and 9377 intensity counts respectively. The spectra FVII (15) and (16), at $882.74 \AA$ and $890.32 \AA$, respectively, are from fluorine, as had been noted in Ref. [13]. This is due to the Teflon material used in multi-channel microwave interferometer. However, it could be also from BII at $882.54 \AA$, due to boron nitride used in Langmuir probe, and FeII at $890.76 \AA$ (vacuum vessel). However, since no other Fe emissions were detected, these emissions are probably from the FVII ion. Other superposed lines are the CIII spectra at $2296.0 \AA$, which is covered by strong NIV*** (71) line, this indicate a leak problem on the TCABR tokamak.

Emissions such as (15) and (16), and also the OIV doublet (11) and (12) are very interesting for spectroscopy purposes, since they have a relative high intensity and are well isolated from other spectral emissions. Moreover, note that identification of spectral emissions with different ionization degrees allows the probing of different regions in the plasma (since more ionized ions are mainly localized inward the plasma). In the wavelength interval presented in Fig. 3, one can identify ions with low ionization potential (localized at colder regions), such as hydrogen (Lyman gamma) with $13.60 \mathrm{eV}$, and CII with $24.38 \mathrm{eV}$, or ions with higher ionization potential, such as OV with $113.87 \mathrm{eV}$, and FVII with $185.18 \mathrm{eV}$ [14].

All these emissions will be seen at higher wavelength as second, third and forth diffraction order emissions with much better spectral resolution, and sometimes superposed with other first line emissions. The appearance of the hydrogen Lyman gamma emission (19) can also be observed in Fig. 3, with a relatively good intensity, but it is affected by the tail of very strong emission CIII (20). Also is important to notice that some spectral emissions as OIV (1) at $554.07 \AA$, OV (9) at $760.35 \AA$ and OIII (13) at 833.51 $\AA$ are sum of many multiplet lines as will be clear when looking at higher diffraction order lines.

In Fig. 4, it is shown emissions from wavelength interval of $1000 \AA$ to $1600 \AA$. The second diffraction order spectra from the 10 first emissions observed in Fig. 1 start to be present. Among the new 9 first diffraction order emis- 


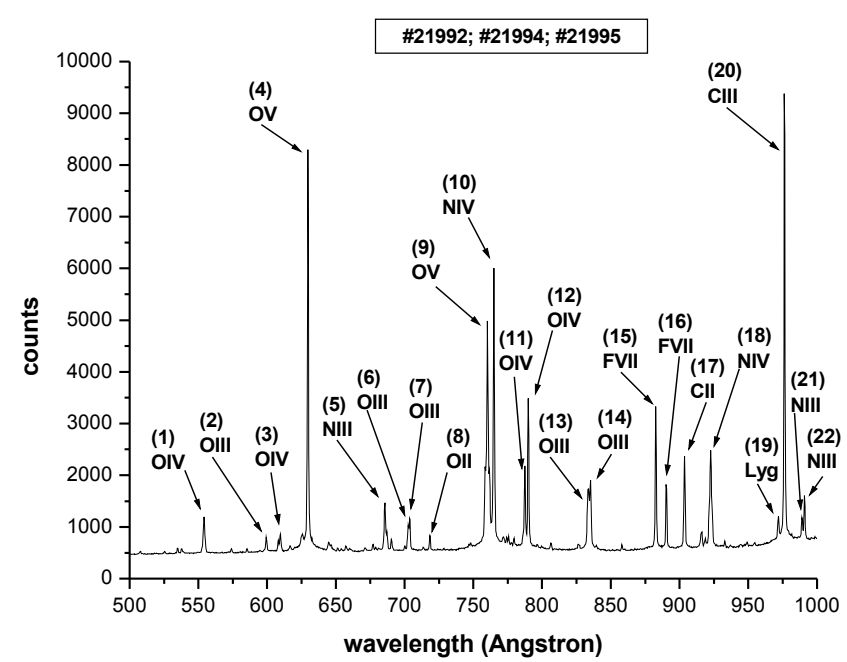

FIG. 3: TCABR VUV spectra from $500 \AA$ to $1000 \AA$ with 22 first diffraction order lines. Important emission lines are: (1), (4), (11), (12), (15), (16), and (20) due to their intensity profile and spectral localization.

sions, one is from a well-isolated single CIII emission, two are from hydrogen (Lyman alpha and beta) and three are doublets (OVI, NV, CIV). The interesting spectral lines are the CIV and NV doublets at $1550.0 \AA$ and $1242.0 \AA$ due to their high spectral intensity and the absence of closer emissions. The OVI doublet at $1032.0 \AA$ is the strongest emission with 38488 counts; however, some care should be taken due to the Lyman beta emission nearby. The Lyman alpha at $1215.0 \AA$, as expected, is the strongest among hydrogen emissions, but notice the presence of a nearby weak OIV second diffraction order line.

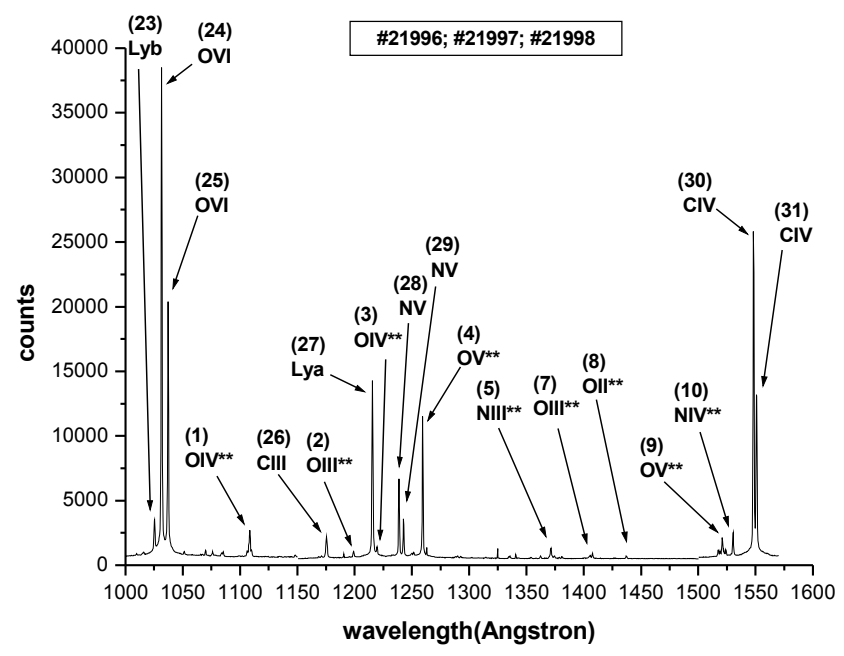

FIG. 4: TCABR VUV spectra from $1000 \AA$ to $1600 \AA$ with 9 first diffraction order and 9 second diffraction order spectra. Emissions from OVI $(24,25)$, Lyman alpha $(27)$, NV $(28,29)$ and CIV $(30,31)$ are the strongest first diffraction order in the spectrum.

Above $1500 \AA$, most emissions are from higher diffraction order spectra. Nevertheless, we still can find some interesting first diffraction order emissions up to $1900 \AA$, as can be seen in Fig. 5. In the emissions from $1550 \AA$ to $2100 \AA$ new 6 first diffraction order lines, 13 second and 2 third diffraction order lines can be observed. In Fig. 5, the doublet emissions from CIV at $1550 \AA$ of Fig. 4 have been omitted in order to be able to work with low intensity emission lines.

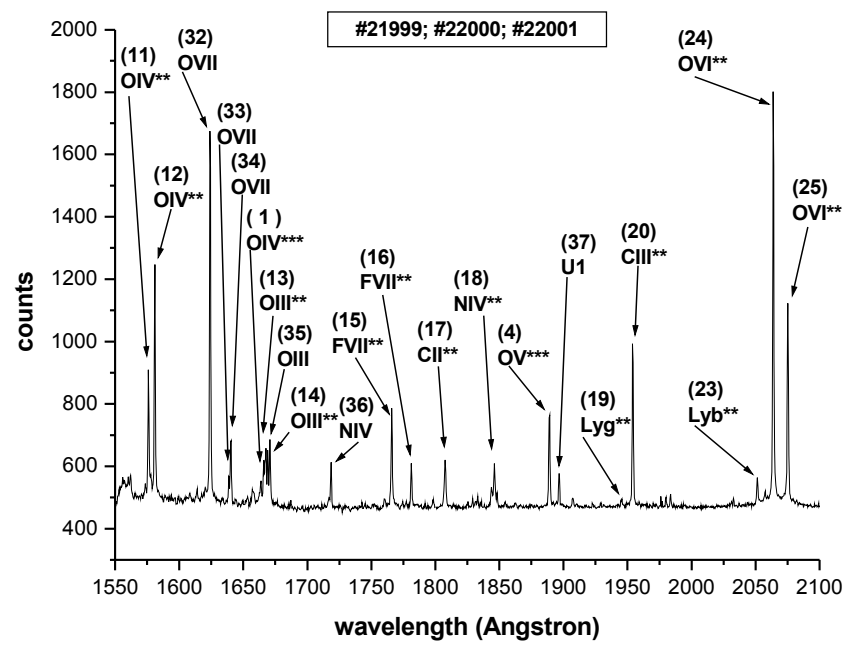

FIG. 5: Emissions from $1550 \AA$ to $2100 \AA$ with 6 first, 13 second and 2 third diffraction order spectra. The U1 (37) line is the last first diffraction order spectra

The OVII (32) emission at $1624.05 \AA$ is a strong line, well isolated, and with high ionization potential $(739.11 \mathrm{eV})$, which makes it interesting for diagnostics. The doublet spectrum of OVII at $1638.74 \AA$ and $1640.18 \AA$ also possesses no nearby lines. The U1 emission (37) at $1896.85 \AA$ has good intensity, is well isolated, but no reference line was found for this emission. In addition, the U1 spectrum is the last first diffraction order emission observed in the measurements. Furthermore, in this interval, some third diffraction order lines are starting to be seen.

In the wavelength interval from $2200 \AA$ to $2600 \AA$ presented in Fig. 6, very low intensity lines are observed, with 4 second, 6 third, and 2 fourth diffraction order emissions. Notice that the highest intensity is lower than 700 counts. The OV line (4) at $2518.95 \AA$ is a well-isolated fourth diffraction order spectrum that can be very useful for the diagnostics. An interesting feature in this interval is that some multiplet emissions can be seen separated with some detail. The OV (1) spectrum, originally at $554.07 \AA$, appears at $2216.27 \AA$ as fourth diffraction order. However, the emission that seemed to be an isolated spectral line in the first diffraction order now splits in three more emissions at $2213.38 \AA, 2218.08 \AA$ and 2220.97 $\AA$. The same behavior is seen for the OV (9) line at $760.35 \AA$ which corresponds to $2281.34 \AA$ at fourth order, with the observation of four other lines at $2275.92 \AA, 2278.45 \AA, 2283.51$ $\AA$, and $2286.04 \AA$. Additionally, OIII (13) at $2501.19 \AA$ also presents multiplet behavior with $2498.30 \AA, 2500.11 \AA$, and $2503.34 \AA$.

In Fig. 7, 2 second order, 9 third order, 2 fourth order and one fifth diffraction order lines are seen. In this interval, many isolated third order diffraction lines, such as FVII (15) and (16), CII (17), CIII (20), can be important for spectroscopic diagnostic. Lyman beta (23) can also be a useful emission. An example of strong overlapping effect of two lines is seen at $3096.00 \AA$. Due to the small spectral difference between 


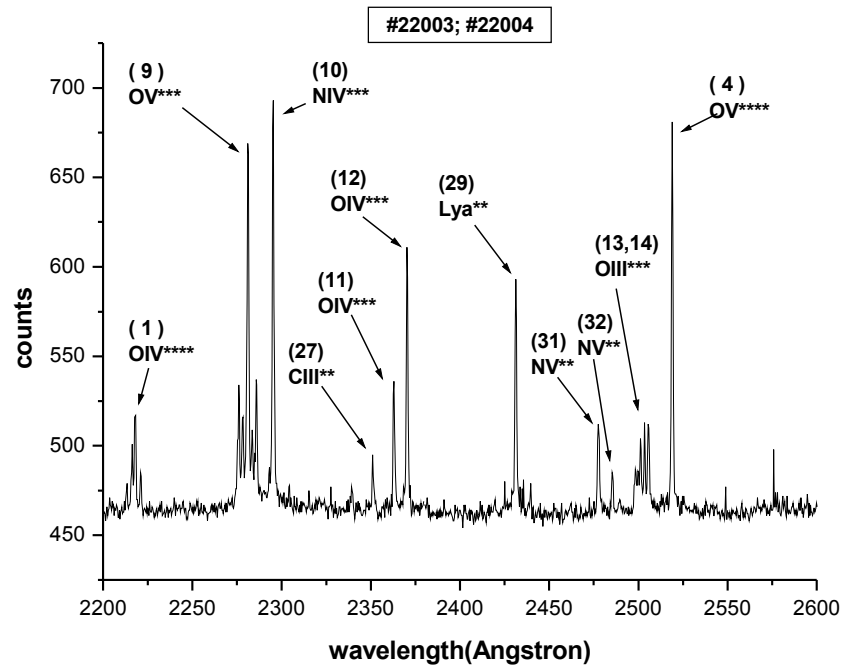

FIG. 6: Emissions from $2200 \AA$ to $2600 \AA$ with 4 second, 6 third, and 2 fourth diffraction order emissions, which corresponds to 13 third and 5 fourth diffraction order multiplet emissions

third order OVI (24) and second order CIV (39), and their strong intensities, overlapping is observed. Finally, the fifth order OV (4) line emission can also be interesting due to its high diffraction order (but care is need to be taken since it seen to be too high intensity compared to fourth diffraction order emission).

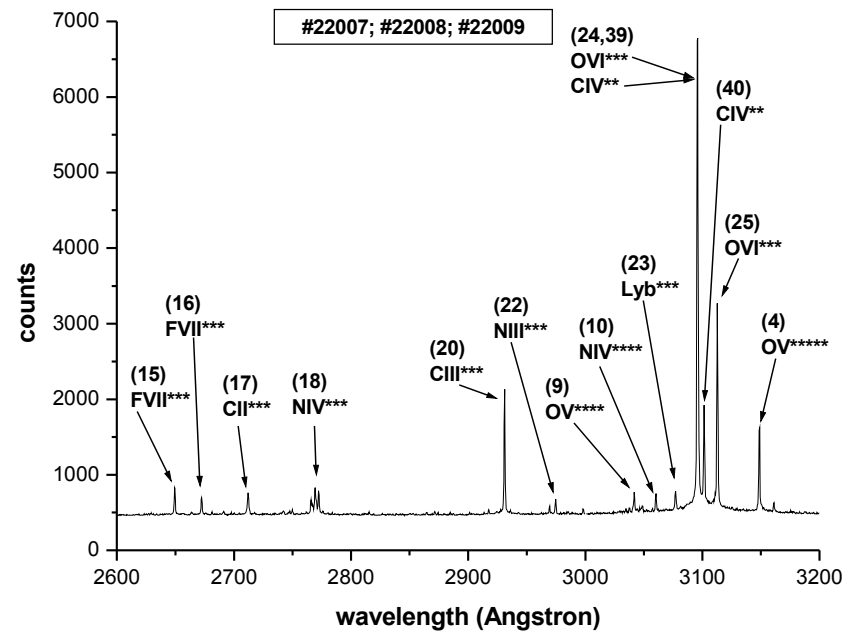

FIG. 7: Emissions from $2600 \AA$ to $3200 \AA$ with 2 second order, 9 third order, 2 fourth order, and one fifth diffraction order emissions

Table 1 lists main emissions presented in Figs. 3 to 7 (including the different diffraction orders observed). All the wavelengths given by $\lambda_{\text {NIST }}$ are from reference [14], and $\lambda_{\text {measured }}$ is the wavelength read directly after the conversion from CCD pixel number. In addition, the last column presents references where similar emissions have been observed $[4$, 13, 15-17].

The use of higher diffraction order lines can be very interesting. As an example, line number 9 in Table $1, \mathrm{OV}$ at 760.35 $\AA$, seen up to fourth diffraction order, is present also in many other larger devices. With configuration $1 s^{2} 2 s 2 p-1 s^{2} 2 p^{2}$, in principle could be a good line for the diagnostic. In Fig.

\begin{tabular}{|c|c|c|c|c|c|}
\hline $\mathbf{N}$ & Spectra & $\begin{array}{c}\lambda_{\text {measured }} \\
(\AA)\end{array}$ & $\begin{array}{c}\lambda_{\text {NIST }} \\
(\AA)\end{array}$ & $\begin{array}{l}\text { Detected Higher } \\
\text { Order number }\end{array}$ & $\begin{array}{l}\text { Observed also } \\
\text { at reference }\end{array}$ \\
\hline 1 & OIV & 554.07 & 554.07 & $2^{\text {nd }}, 3^{\text {rd }}, 4^{\text {th }}$ & {$[4,13,15]$} \\
\hline 2 & OIII & 599.40 & 599.60 & $2^{\text {nd }}$ & \\
\hline 3 & OIV & 609.75 & 609.83 & $2^{\text {nd }}$ & \\
\hline 4 & $\mathrm{OV}$ & 629.73 & 629.73 & $2^{\text {nd }}, 3^{\text {rd }}, 4^{\text {th }}, 5^{\text {th }}$ & {$[4,13,15,16,17]$} \\
\hline 5 & NIII & 685.76 & 685.82 & $2^{\text {nd }}$ & \\
\hline 6 & OIII & 702.53 & 702.33 & & [4] \\
\hline 7 & OIII & 703.61 & 703.85 & $2^{\text {nd }}$ & [4] \\
\hline 8 & OII & 718.24 & 718.50 & $2^{\text {nd }}$ & \\
\hline 9 & $\mathrm{OV}$ & 760.35 & 760.45 & $2^{\text {nd }}, 3^{\text {rd }}, 4^{\text {th }}$ & {$[13,15,16,17]$} \\
\hline 10 & NIV & 764.99 & 765.15 & $2^{\text {nd }}, 3^{\text {rd }}, 4^{\text {th }}$ & {$[17]$} \\
\hline 11 & OIV & 787.47 & 787.71 & $2^{\text {nd }}, 3^{\text {rd }}, 4^{\text {th }}$ & [16] \\
\hline 12 & OIV & 789.97 & 790.11 & $2^{\text {nd }}, 3^{\text {rd }}$ & {$[16]$} \\
\hline 13 & OIII & 833.51 & 833.74 & $2^{\text {nd }}, 3^{\text {rd }}$ & {$[16,17]$} \\
\hline 14 & OIII & 835.29 & 835.29 & $2^{\text {nd }}, 3^{\text {rd }}$ & {$[16,17]$} \\
\hline 15 & FVII & 882.74 & 883.10 & $2^{\text {nd }}, 3^{\text {rd }}$ & [13] \\
\hline 16 & FVII & 890.23 & 890.76 & $2^{\text {nd }}, 3^{\text {rd }}$ & [13] \\
\hline 17 & $\mathrm{CII}$ & 903.79 & 903.62 & $2^{\text {nd }}, 3^{\text {rd }}$ & {$[4,16]$} \\
\hline 18 & NIV & 922.70 & 922.52 & $2^{\text {nd }}, 3^{\text {rd }}$ & {$[17]$} \\
\hline 19 & Lgama & 971.96 & 972.54 & $2^{\text {nd }}$ & \\
\hline 20 & CIII & 976.24 & 977.03 & $2^{\text {nd }}, 3^{\text {rd }}$ & {$[4,13,16]$} \\
\hline 21 & NIII & 989.09 & 989.79 & & {$[17]$} \\
\hline 22 & NIII & 990.87 & 991.51 & $2^{\text {nd }}, 3^{\text {rd }}$ & [17] \\
\hline 23 & Lbeta & 1025.46 & 1025.72 & $2^{\text {nd }}, 3^{\text {rd }}$ & \\
\hline 24 & OVI & 1031.66 & 1031.91 & $2^{\text {nd }}, 3^{\text {rd }}$ & {$[4,13,15,17]$} \\
\hline 25 & OVI & 1037.28 & 1037.61 & $2^{\text {nd }}, 3^{\text {rd }}$ & {$[4,13,15,17]$} \\
\hline 26 & CIII & 1175.49 & 1175.26 & $2^{\text {nd }}$ & {$[4,13]$} \\
\hline 27 & Lalpha & 1215.68 & 1215.67 & $2^{\text {nd }}$ & {$[4,17]$} \\
\hline 28 & $\mathrm{NV}$ & 1238.65 & 1238.82 & $2^{\text {nd }}$ & {$[17]$} \\
\hline 29 & NV & 1242.59 & 1242.80 & $2^{\text {nd }}$ & {$[17]$} \\
\hline 30 & CIV & 1548.19 & 1548.19 & $2^{\text {nd }}$ & {$[4,17]$} \\
\hline 31 & CIV & 1550.70 & 1550.77 & $2^{\text {nd }}$ & {$[4,17]$} \\
\hline 32 & OVII & 1624.05 & 1623.64 & & \\
\hline 33 & OVII & 1638.74 & 1638.27 & & \\
\hline 34 & OVII & 1640.18 & 1639.88 & & \\
\hline 35 & OIII & 1669.21 & 1669.31 & & \\
\hline 36 & NIV & 1718.66 & 1718.55 & & \\
\hline 37 & $\mathrm{U}_{1}$ & 1896.85 & Unknown & & \\
\hline
\end{tabular}

Table 1: List of most intense line emissions observed from $55 \mathrm{~nm}$ to $320 \mathrm{~nm}$, including the measured species, measured wavelengths, wavelengths from Ref [14], detected diffraction order numbers, and other references in the literature

$8 \mathrm{a}$, the first diffraction order is seen, with other emissions on neighborhood but no clear separation. When third diffraction order is observed, as in Fig 8b, it is clear that there are five spectral lines. According to Ref. [14] these lines corresponds to main central line at $760.446 \AA$, one doublet at 759.442 $\AA$ and $761.128 \AA$, and another doublet lines at $758.677 \AA$ and $762.004 \AA$. So, the correct separation of these spectral lines can only be seen in the present experiment if the higher diffraction order lines are used.

Another very important issue is the care that must be taken about CCD pixel distribution on the spectral profiles during the measurements. The first, fourth and fifth diffraction order of the well known OV line (4) at $629.73 \AA$ are shown in Fig. 9.

Taking the oxygen mass value and Gaussian profile FWHM (full width at half maximum), we obtain the following ion temperatures for each diffraction order: $870 \mathrm{eV}, 109 \mathrm{eV}$ and $36 \mathrm{eV}$. The ion temperature obtained from first diffraction order is too high for our tokamak, and this is due to large error that exists when the spectrometer instrumental broadening is larger than the Doppler broadening (confirming the importance of high order diffraction emissions). The result from fourth diffraction order is the correct value, and it has been confirmed from other line measurements. Measurements using emissions such as CIII line at $976.39 \AA$ and OVI line at 1037.21 $\AA$, also presented results around $100 \mathrm{eV}$. On another 

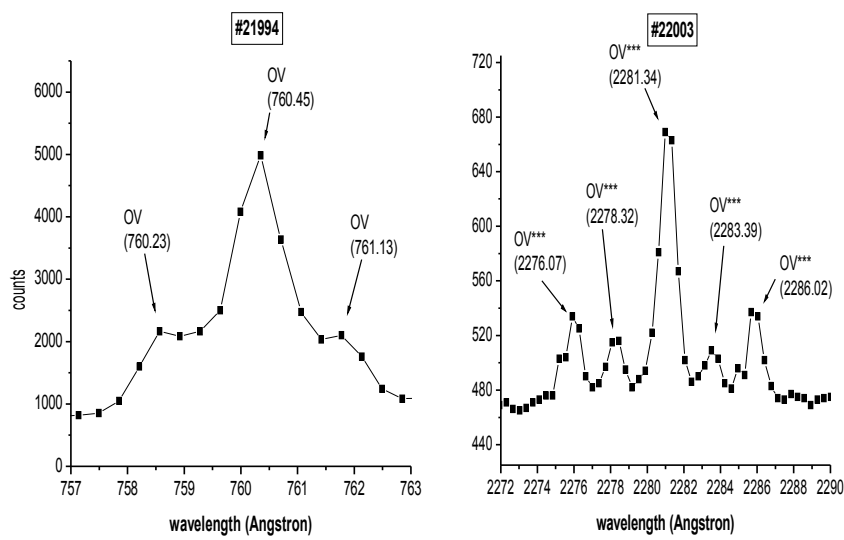

FIG. 8: a) Line OV at $760.45 \AA$ seen at first order. b) OV seen at third order at $2281.34 \AA$
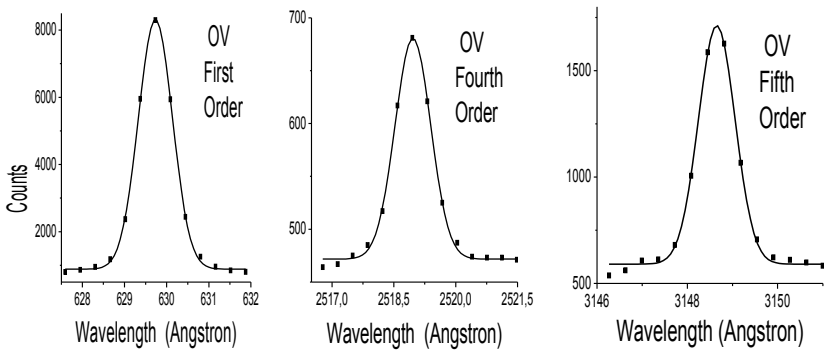

FIG. 9: OV (a) first, (b) fourth and (c) fifth diffraction order emission at $62.973 \mathrm{~nm}, 251.895 \mathrm{~nm}$, and at $314.881 \mathrm{~nm}$ respectively, with their Gaussian fittings hand, the L $\alpha$ line at $1215.58 \AA$ measurement lead to ion temperature of $23 \mathrm{eV}$, since this emission is originated from the outer plasma region. Therefore, the value obtained from fifth diffraction order is too low to be true, this is due to nonsymmetrical pixel distribution on this line compared to the other two lines [18]. So, the ion temperature measurement using different line emissions (from similar ionization stage) helps to check the accuracy of each diffraction order used.

\section{CONCLUSION}

We show that the VUV region of tokamak plasma light emission is very rich of impurity spectral light emissions. Very broad impurity ionization stages are met, such as OII to OVII. We obtained and catalogued 37 first diffraction order lines, which resulted in 28 second, 24 third and 7 fourth diffraction order lines in the interval of $500 \AA$ to $3200 \AA$ VUV region. Most of these detected emissions are observed in other high temperature tokamak plasmas, showing the usefulness of this catalogue. The higher diffraction order lines have shown to be much better to analyze the multiplet structure of the line emissions. Also, the ion temperature measurements are much more accurate if higher diffraction order lines are used, due to the larger Gaussian FWHM value. Moreover, the effect of differences on pixel distribution throughout Gaussian profile showed to produce errors on the ion temperature determination.

\section{ACKNOWLEDGMENTS}

This work has been supported by FAPESP, CNPq, CNEN and FAEPEX/UNICAMP.
[1] A. Manini et al., and the ASDEX Upgrade Team, Nucl. Fusion 46, 1047 (2006)

[2] P. C. de Vries et al., Plasma Phys. Control. Fusion 48, 1693 (2006).

[3] R. Katai et al., Rev. Sci. Instrum. 77, 10F307 (2006).

[4] H. Kubo et. al., Nucl. Fusion 33, 427, 1993.

[5] J. Gafert et al., and ASDEX Upgrade Team, Plasma Phys. Control. Fusion 39, 1981 (1997).

[6] I.C. Nascimento et al., Nucl. Fusion 45, 796, (2005).

[7] H.Sakakita et al., Rev. Sci. Instrum. 74, 2111, (2003).

[8] R.B. Gomes et. Al., Rev. Sci. Instrum. 74, 2071 (2003).

[9] A.M. Daltrini and M. Machida, IEEE Trans. Plasma Science 33, 1961 (2005)

[10] A.M. Daltrini and M. Machida, Rev. Sci. Instrum. 78, 066101, (2007).
[11] A.M. Daltrini et al., Braz. J, Phys. 31, 496, (2001).

[12] A.M. Daltrini and M. Machida, Rev. Sci. Instrum. 76, 053508, (2005).

[13] R.C. Isler, Fusion Enginnering and Design 34-35, 115, (1997).

[14] http://www.physics.nist.gov/cgi-bin/ASD/

[15] W. Biel, G. Bertschinger, and the TEXTOR Team, Rev. Sci. Instrum. 57, 2471, (2004).

[16] A.R. Field et al., Rev. Sci. Instrum. 66, 5433, (1995).

[17] V. Piffl and V. Weinzettl, 29th EPS Conference on Plasma Phys. and Contr. Fusion, 26B, P-4.123, (2002).

[18] M. Machida et al., to be published in the Journal of Plasma and Fusion Research Series - Japan Society of Plasma Science, JSPF, 2009. 